\title{
Topical Ganciclovir in Herpetic Eye Disease
}

\author{
Khalid F Tabbara

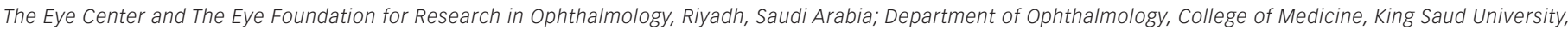 \\ Riyadh, Saudi Arabia; The Wilmer Ophthalmological Institute of The Johns Hopkins University School of Medicine, Baltimore, Maryland, US.
}

DOI: $h$ ttp://doi.org/10.17925/USOR.2016.09.01.25

\begin{abstract}
Herpetic keratitis is a common cause of ocular morbidity and corneal blindness. The disease has a worldwide distribution. There are different types of Herpes virus with variable ocular and adnexal manifestations. Herpes virus stays dormant in the nerve ganglia following a primary infection. Topical antiviral agents include acyclovir and ganciclovir. Both agents may be used for the treatment and prophylaxis of herpetic keratitis. Antiviral prophylaxis is of crucial importance in the prevention of recurrent keratitis in patients with penetrating keratoplasty for herpes induced corneal scars. Recurrent herpetic keratitis in such patients frequently eventuate in the rejection of the graft. This article reviews topical ganciclovir $0.15 \%$ gel in the therapy and prophylaxis of herpetic keratitis.
\end{abstract}

\section{Keywords}

Acyclovir, antiviral therapy, corneal epithelial keratitis, ganciclovir, herpes virus, herpes virus 1, herpes virus 2, herpetic keratitis, stromal keratitis

Disclosure: Khalid F Tabbara has nothing to declare in relation to this article. No funding was received in the publication of this article. Acknowlegements: This Study was presented in part at the Annual meeting of the American Academy of Ophthalmology (AAO), 14-17 November 2015, Sands Expo and Convention Centre, Las Vegas, Nevada, US.

Open Access: This article is published under the Creative Commons Attribution Noncommercial License, which permits any noncommercial use, distribution, adaptation, and reproduction provided the original author(s) and source are given appropriate credit.

Received: February 4, 2016 Accepted: March 8, 2016 Citation: US Ophthalmic Review, 2016;9(1):25-9

Correspondence: Khalid F Tabbara, The Eye Center, 241 Makkah Road, PO Box 55307, Riyadh 11534, Saudi Arabia. E: k.tabbara@nesma.net.sa

Herpetic keratitis is the most common cause of corneal blindness, and is considered to be a major indication for penetrating keratoplasty (PKP). ${ }^{1}$ Herpetic keratitis is caused by human herpes virus (HHV). Table 1 shows the herpes viruses that may cause disease in man. ${ }^{2-5}$

Certain viral infections have been eradicated by vaccination, and others are controlled by public health measures. The resilience of viruses has led to the emergence of new viruses and the evolution of old viruses causing potentially blinding diseases of the eye. HHV type 1 (HHV-1) is endemic worldwide and humans are the only known natural reservoir for the virus. By age 60 , approximately $95 \%$ of individuals are infected by HHV-1. HHV-1 is transmitted by direct contact with infected secretions such as saliva or tears or coming in contact with cutaneous lesions infected by HHV-1.There are approximately one million cases of new ocular herpetic infections annually worldwide.

Patients make an average of four visits to the ophthalmologist for the first episode and six visits for recurring episodes of herpetic keratitis. ${ }^{6-10}$ It is estimated that close to two-thirds of all cases of primary herpes infections are asymptomatic. Initial exposure to HHV leads to primary infection. The virus becomes latent in the nerve ganglia and approximately $98 \%$ of patients with herpes infection shed HHV-1 genome in tears or saliva. ${ }^{11}$ Following a primary infection, the virus stays latent in the sensory and autonomic neurons of ganglia (trigeminal ganglion, ciliary ganglion, superior cervical ganglion). The recurrences (secondary infection) of herpetic lesions are the result of reactivation of the virus.6,12,13 The reactivation leads to shedding of the virus or recurrence of the disease. There are risk factors both genetic and environmental that lead to reactivation. The clinical manifestations depend on the site of latency. Infection with HHV is determined by local susceptibility, general host immunity, the virus strain, and genetic predisposition. ${ }^{14}$ The site of viral latency determines the future of reactivation and clinical manifestations of the disease. Latency of HHV in the trigeminal ganglion may lead to recurrence of herpetic keratitis. There are 25 different types of neurons in the trigeminal ganglion.

Latency in the superior cervical ganglion may lead to reactivation and recurrence of herpetic intraocular inflammation and anterior uveitis..$^{12,13}$ Latency in the ciliary ganglion may lead to recurrent intraocular inflammation. Alpha adrenergic compounds and estradiol may lead to the reactivation of HHV from the neurons of the trigeminal ganglia. Infection of the autonomic nervous system and latency in the superior cervical ganglion may lead to uveitis but not keratitis. On the other hand, latency in the lateral geniculate body may lead to acute retinal necrosis.

Systemic and intravitreal administration of ganciclovir have been recommended for the treatment of cytomegalovirus and retinitis and for 
Table 1: Herpes Virus Types Causing Disease in Humans ${ }^{2-5}$

\begin{tabular}{|c|c|c|c|c|}
\hline Herpes virus & Primary infection & Site of latency & Secondary recurrent infectio & Therapy \\
\hline HHV-1 (Alpha) & Orolabial, ocular & Cranial sensory ganglia & $\begin{array}{l}\text { Herpes labialis keratitis } \\
\text { intraocular inflammation }\end{array}$ & Acyclovir/Ganciclovir \\
\hline HHV-2 (Alpha) & Genital, perinatal & $\begin{array}{l}\text { Lumbar and sacral } \\
\text { Sensory ganglia }\end{array}$ & Dermatitis vaginitis & Acyclovir/Ganciclovir \\
\hline $\begin{array}{l}\text { HHV-3 (Alpha) } \\
\text { (Varicella zoster) }\end{array}$ & $\begin{array}{l}\text { Lungs, skin, } \\
\text { Disseminated varicella }\end{array}$ & $\begin{array}{l}\text { Cranial and thoracic } \\
\text { Sensory ganglia }\end{array}$ & Varicella zoster & $\begin{array}{l}\text { Acyclovir/Valcyclovir/Foscarnet } \\
\text { Vaccine available }\end{array}$ \\
\hline $\begin{array}{l}\text { HHV-4 (Gamma) } \\
\text { (Epstein-Barr virus) }\end{array}$ & Infectious mononucleosis & B cells & Unknown & None \\
\hline HHV-5 (Cytomegalovirus) (Beta) & Disseminated, congenital & Variety of tissues & Immunocompromised patients & Ganciclovir/Foscarnet \\
\hline HHV-6 (Beta) (6A, 6B) & $\begin{array}{l}\text { Exanthema subitum } \\
\text { (Roseola infantum) }\end{array}$ & Salivary glands & CNS & None \\
\hline HHV-7 (Beta) & $\begin{array}{l}\text { Exanthema subitum } \\
\text { (Roseola infantum) }\end{array}$ & Salivary glands & Unknown & None \\
\hline HHV-8 (Gamma) & Skin, conjunctiva & Unknown & Kaposi sarcoma & Interferon-alpha 2b \\
\hline
\end{tabular}

the treatment of herpetic acute retinal necrosis in immunocompetent and immunocompromised individuals.

\section{Objective}

The main objective of this review is to elucidate topical ganciclovir $0.15 \%$ gel in the therapy and prophylaxis of herpetic keratitis.

\section{Human Herpes Virus}

$\mathrm{HHV}-1$ is the most commonly encountered virus in herpetic keratitis. The word "herpes" is derived from the Greek word "herpein" or "to creep" because of the characteristic cutaneous eruption. HHV is a doublestranded DNA virus consisting of 12 pentavalent and 150 hexavalent capsomeres measuring $110 \mathrm{~nm}$. The DNA virus is in proteinaceous matrix and has a lipid envelope measuring $220 \mathrm{~nm} .{ }^{4} \mathrm{HHV}$ may affect any of the ocular structures or adnaxae. HHV is a great mimicker of other microbial keratitis cases. Ocular manifestations may include blepharitis, conjunctivitis, keratitis, optic neuritis, uveitis, retinitis, and trabeculitis. By far the most common manifestation of herpetic disease of the eye is keratitis. The keratitis may be epithelial, stromal, or endothelial. HHV may affect immunocompetent and immunocompromised hosts.

Three of the eight major herpes viruses are oncogenic. HHV-4 or EpsteinAchong-Barr virus may cause lymphoma. HHV-8 may cause Kaposi's sarcoma in patients with human immune-deficiency viral (HIV) infection. Cytomegalovirus has been associated with mucoepidermoid carcinoma. ${ }^{5}$

\section{Antiviral Agents}

Over the past 30 years, the United States Food and Drug Administration (FDA) has approved a limited number of topical antiviral agents. The only two topical antiviral agents approved in the United States are ganciclovir and trifluoridine. Although topical acyclovir is used globally, it is not approved in the United States for herpetic keratitis. Topical idoxuridine and vidarabine were previously available but currently are not available for clinical use. Oral agents that are available for the treatment of herpetic eye disease include acyclovir, ganciclovir, valaciclovir, valganciclovir, famciclovir, and foscarnet. Oral antiviral agents may be used in patients who suffer from arthritis or hand tremors and are unable to use topical ointments, gels or eye drops. Oral antiviral agents may be used in patients who have to wear contact lenses and in pediatric patients who are refractory to topical antiviral agents. Patients who require long term treatment may need systemic antivirals. Oral antivirals may also be used in patients who require prophylaxis following ocular surgery. Topical antivirals are safe and effective in epithelial keratitis but adjunctive systemic therapy may be required in stromal keratitis, uveitis, and in patients with keratouveitis.

Topical antiviral therapy is preferred in patients with renal impairment, elderly patients, pregnant patients, and nursing mothers. Systemic ganciclovir, on the other hand, may lead to bone marrow suppression. Longterm oral acyclovir is not a recommended prophylaxis because of potential nephrotoxicity. Topical ganciclovir may provide adequate antiviral levels in the aqueous humor sparing systemic toxicity. ${ }^{14}$ Future prospective clinical trials are needed to assess the safety and efficacy of topical antivirals versus oral antivirals in the prevention of recurrent herpetic keratitis.

The optimal dosage of the antiviral prophylactic agents should be determined.

In patients who undergo PKP for herpetic corneal scars, the rate of recurrences without prophylaxis may be high. Oral antivirals in herpetic keratitis are used for the prevention of HHV recurrences..$^{15}$ They are potentially nephrotoxic, the cost is high, they have limited ocular tissue absorption, cannot be used in pregnancy, and cannot be used long-term.

Topical ganciclovir $0.15 \%$ gel (Zirgan ${ }^{\circledR}$, Bausch + Lomb, USA) is a potent inhibitor of members of the herpes family. The drug can reach therapeutic levels for herpes virus in the cornea and aqueous humor following topical application. ${ }^{14}$ Topical ganciclovir $0.15 \%$ gel induces rapid apoptosis of herpes infected epithelial cells in the cornea and induces rapid healing of the herpetic keratitis. In addition, ganciclovir can induce preferential distribution in melanin-bearing cells. This binding of ganciclovir to the melanin is desirable in patients who suffer from herpetic anterior uveitis and iritis, providing a reservoir and prolonged delivery of the antiviral agent. 
Topical ganciclovir gel in herpetic keratitis has no ocular surface complications, no hematologic side-effects, and does not reach to toxic levels in the blood stream following topical application, and, therefore, does not lead to nephrotoxicity or bone marrow suppression.

In patients with corneal scars due to HHV infection and undergoing PKP, it is recommended that they are placed on prophylactic topical ganciclovir to prevent recurrences of herpetic keratitis in the corneal graft and subsequent corneal rejection. Several antiviral agents can be ineffective due to resistance of the virus, causing it to recur mainly in immunocompromised patients and sometimes in immunocompetent patients.

\section{Topical Ganciclovir}

Ganciclovir inhibits DNA polymerase and interferes with DNA synthesis Topical ganciclovir $0.15 \%$ gel was evaluated in the treatment of herpetic keratitis. ${ }^{16-19}$ A total of 16 consecutive patients with herpetic keratitis were included in a non-randomized prospective, interventional study. There were 12 male and 4 female patients with herpetic epithelial keratitis (see Figures 1-3).

The age range was $26-80$ years (mean age: 47 years). Ten cases had dendritic keratitis and six patients had recurrent geographic epithelial keratitis. Complete resolution of the herpetic keratitis was noted in all patients. The corneal lesions in 11 (69\%) of the 16 patients healed within 7 days, and 5 (31\%) patients healed within 3 weeks..$^{18}$ There were no ocular side effects from the topical use of ganciclovir noted among the 16 patients. The effect of ganciclovir in the treatment of herpetic epithelial keratitis was compared to acyclovir 3\% ointment. Topical ganciclovir and topical acyclovir were equally effective in the therapy of herpetic epithelial keratitis. ${ }^{17}$

In our experience, topical use of ganciclovir in adenoviral keratoconjunctivitis has limited efficacy and does not prevent subepithelial corneal opacities following adenoviral keratoconjunctivitis.

\section{Ganciclovir Prophylaxis Following Penetrating Keratoplasty}

The topical use of corticosteroids helps in the prevention of corneal graft rejection but at the same time increases the susceptibility of recurrences of herpetic keratitis. The recurrences of herpetic keratitis may lead to corneal graft rejection. We studied the effects of topical ganciclovir $0.15 \%$ gel in the prophylaxis of herpetic epithelial keratitis following PKP.

Prophylactic topical ganciclovir may lead to prevention of recurrent herpetic keratitis following PKP for patients with corneal scars secondary to previous herpetic keratitis. ${ }^{18}$ Topical ganciclovir appears to be more effective than oral acyclovir in the prevention of recurrences of herpetic keratitis following PKP (Figures $4 a, 4 b) .{ }^{18}$ Topical ganciclovir in herpetic keratitis is a helpful therapeutic modality for the management of recurrent herpetic epithelial keratitis and in the prophylaxis of patients with herpetic keratitis following PKP (Figure 5).

Topical ganciclovir has no significant systemic absorption, and therefore no hematologic abnormalities are noted. The gel is easy to apply, does not cause blurring or irritation, is well tolerated, and has no toxic effect on the ocular surface. Ganciclovir $0.15 \%$ gel has potential role in the prophylaxis of patients with herpetic keratitis undergoing PKP.
Figure 1: Shows a 32-year-old Female Patient with a Large Geographic Corneal Ulcer Caused by Human Herpes Virus type 1

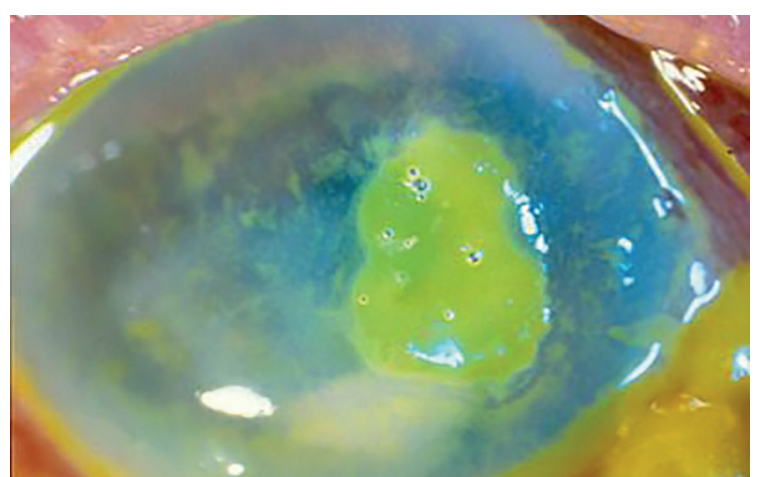

Figure 2: Multi-nucleated Epithelial Cell in the Corneal Scrapings of the Patient Shown in Figure 1

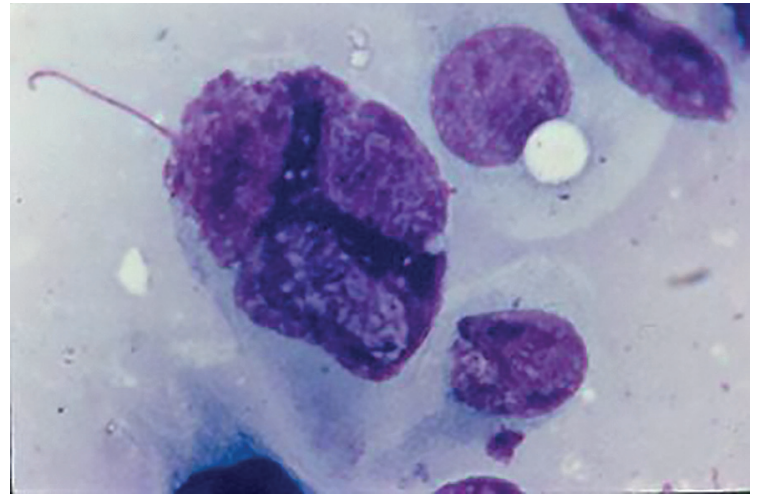

Figure 3: Herpetic Epithelial Keratitis in a 67-year-old Male

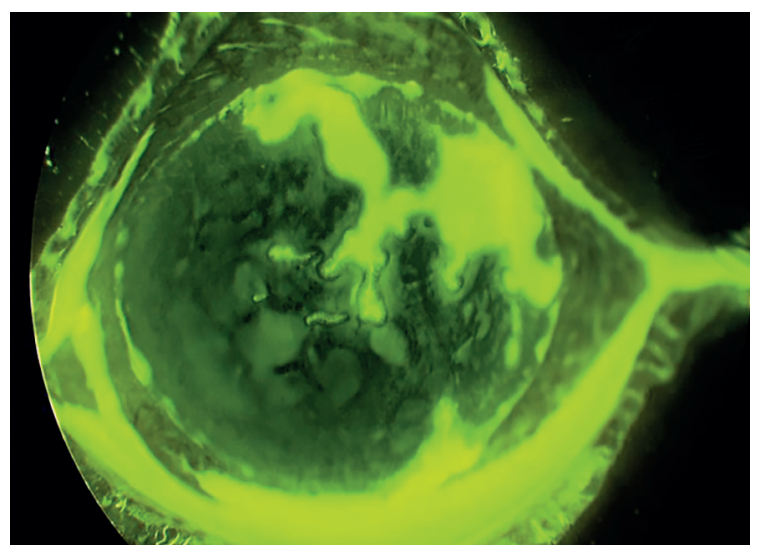

The main indication for therapy of recurrent herpetic keratitis with topical ganciclovir $0.15 \%$ gel is to relieve the discomfort, prevent sight threatening complications, and prevent recurrences induced by the prolonged use of topical steroids use after PKP.

The Herpetic Eye Disease Study (HDES) ${ }^{9}$ was a multicenter, randomized trial which enrolled 703 patients. It focused on the use of oral acyclovir to prevent 
A

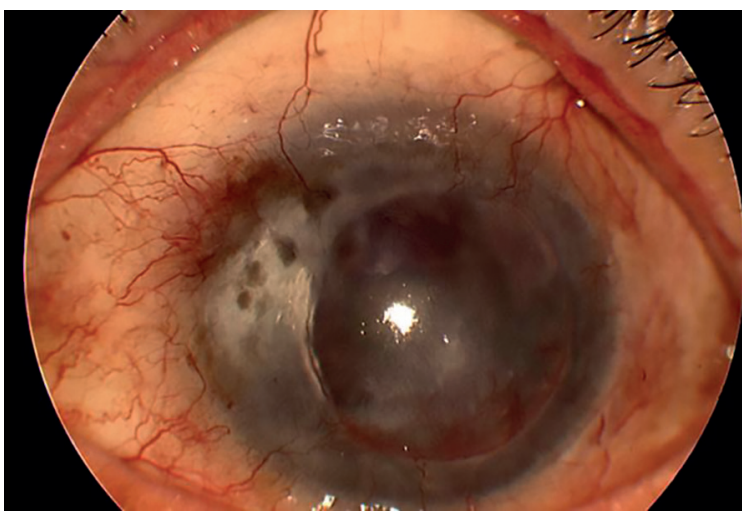

B

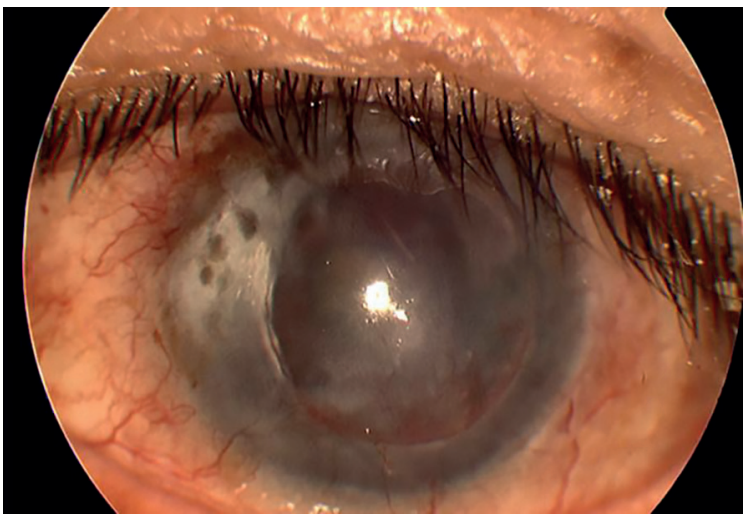

Figure 5: Failed Corneal Graft in a Patient with Herpetic Keratitis who Underwent Penetrating Keratoplasty and had Recurrence of Herpetic Keratitis

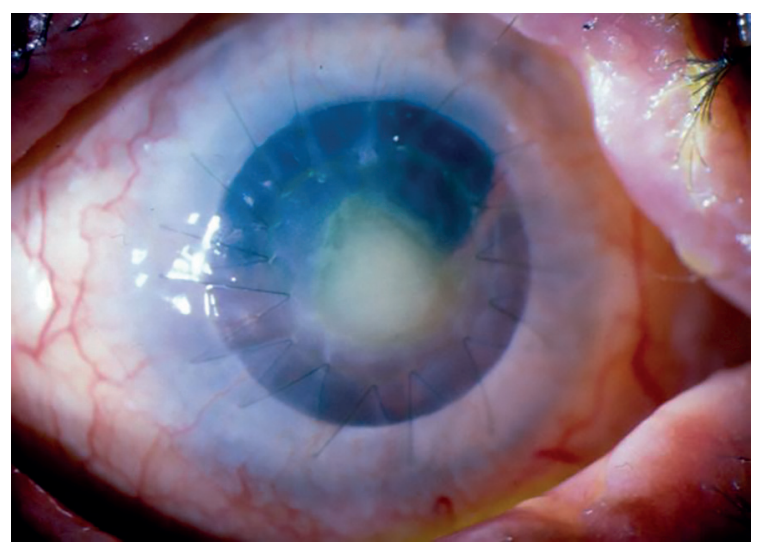

herpetic epithelial and stromal keratitis, and determined the recurrence rate following prophylaxis with oral acyclovir. ${ }^{8}$ Each patient included in the study had at least one episode of ocular herpes virus in the previous 12 months and no disease activity within the previous 30 days. ${ }^{9}$ Patients were assigned to receive either $400 \mathrm{mg}$ acyclovir twice daily or placebo. ${ }^{9}$ Patients were examined at day $1,3,6,9$, and 12 months after the start of treatment. Recurrences of ocular herpes in the placebo group was $32 \%$ in the 12-month period. Starting with the cohort of patients with history of some form of ocular herpes, the rate of herpetic epithelial keratitis recurrences
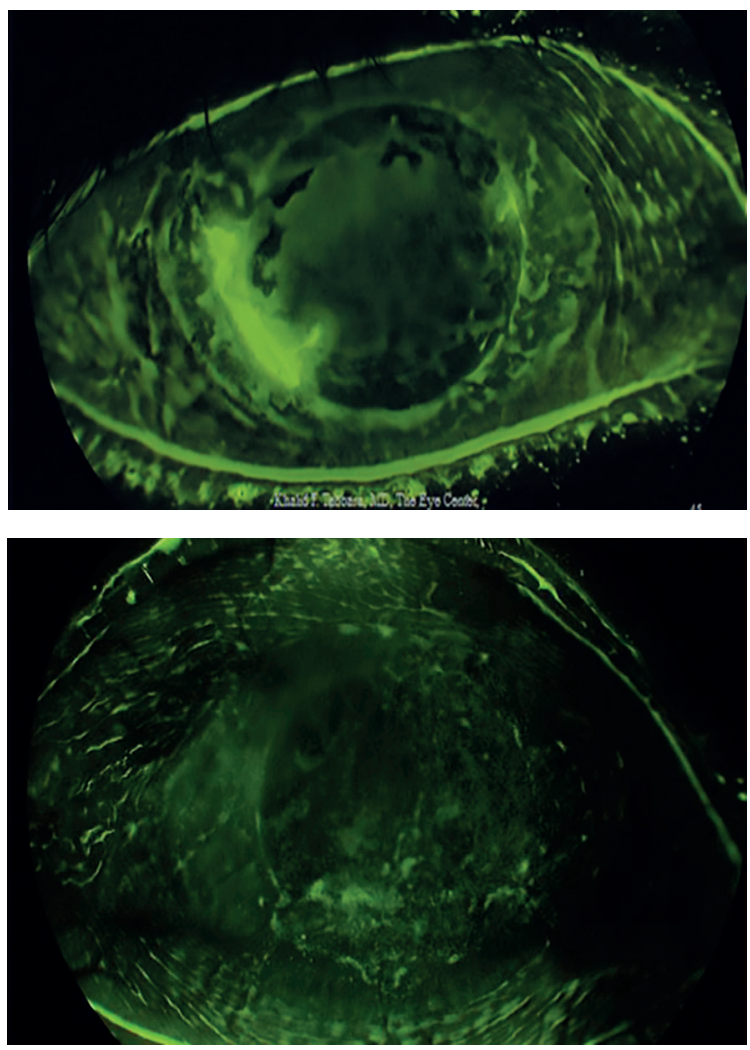

following acyclovir treatment or placebo were essentially similar $12 \%$ and $15 \%$ respectively. There was no statistically significant difference in the recurrences of epithelial keratitis between those who received prophylactic oral acyclovir or placebo. The recurrence rate of herpetic stromal keratitis was different. Three percent of patients with history of ocular herpes but not stromal keratitis developed herpetic stromal keratitis compared to $28 \%$ of patients with positive history of stromal keratitis. Patients who had higher number of previous episodes of stromal keratitis had increased risk of future recurrences.

Wang and associate ${ }^{20}$ studied oral ganciclovir in the treatment of herpes simplex keratitis. In a randomized controlled single-blind prospective study, the authors included 60 patients with herpetic stromal keratitis and corneal endothelialitis. Patients with corneal graft required the topical use of corticosteroids in the form of prednisolone acetate $1 \%$. The initial dosage was every two hours for one week, followed up by four times daily. The authors found out that short term oral acyclovir induced healing, shortened the course, and reduced the recurrences rate of patients with herpetic keratitis and endothelialitis. New prodrugs that inhibit thymidine kinase are being developed and are promising for the clinical use in the near future. ${ }^{21}$

\section{Conclusion}

Herpetic keratitis is a potentially blinding disease that requires frequent visits to the ophthalmologist and is responsible for the significant socioeconomic loss and waste of productivity. Ganciclovir helps in the treatment and prevention of recurrent herpetic keratitis in patients undergoing PKP for corneal scars caused by HHV. 
1. Wilhelmus KR, Epidemiology of Ocular Infections In. Tasman W. (ed). Duane's Foundation of Clinical Ophthalmology Philadelphia, US: Lippincott Williams \& Wilkins, 1992.

2. Drew WL. In. Herpes Viruses. Ryan KJ, Ray CG (eds). An Introduction to Infectious Diseases. Sherris Medical Microbiology 4th ed, Ohio, US: McGraw-Hill Medical Publishing Division, 2004, pg 555-576.

3. Adams, MJ, Carstens EB, Ratification vote on taxonomic proposals to the International Committee on Taxonomy of Viruses (2012), Arch Virol, 2012;157: 1411-22.

4. Whitley RJ, Herpesviruses. In: Baron S, Baron's Medical Microbiology 4th ed, Galveston, Texas: Univ of Texas Medical Branch, 1996.

5. Murray PR, Rosenthal KS, Pfaller MA, Medical Microbiology 5th ed, Missouri, US: Elsevier Mosby, 2005.

6. Pires de Mello CP, Bloom DC, Paixão IC, Herpes simplex virus type-1: replication, latency, reactivation and its antiviral targets, Antivir Ther, 2016 [Epub ahead of print].

7. Young RC, Hodge DO, Liesegang TJ, Baratz KH, Incidence, recurrence, and outcomes of herpes simplex virus eye disease in Olmsted County, Minnesota, 1976-2007: the effect of oral antiviral prophylaxis, Arch Ophthalmol, 2010:128:1178-83.
8. Herpetic Eye Disease Study Group, Oral acyclovir for herpes simplex virus eye disease: effect on prevention of epithelial keratitis and stromal keratitis. Herpetic Eye Disease Study Group, Arch Ophthalmol, 2000:118:1030-6.

9. Wilhelmus KR, Beck RW, Moke PS, et al., Acyclovir for the Prevention of Recurrent Herpes Simplex Virus Eye Disease. Herpetic Eye Disease Study Group, N Eng/ I Med, 1998;339:300306

10. Liesegang $\mathrm{TJ}$, Classification of herpes simplex virus keratitis and anterior uveitis, Cornea, 1999;18:127-43.

11. Kaufman HE, Azcuy AM, Varnell ED, et al., HSV-1 DNA in tears and saliva of normal adults, Invest Ophthalmol Vis Sci, 2005;46:241-7.

12. Nagel MA, Rempel A, Huntington J, et al., Frequency and abundance of alphaherpesvirus DNA in human thoracic sympathetic ganglia, I Virol, 2014;88:8189-92

13. Lee S, Ives AM, Bertke AS, Herpes simplex virus 1 reactivates from autonomic ciliary ganglia independently from sensory trigeminal ganglia to cause recurrent ocular disease, J Virol, 2015;89:8383-91.

14. Castela N, Vermerie N, Chast F, et al., Ganciclovir ophthalmic ge in herpes simplex virus rabbit keratitis: intraocular penetration and efficacy, J Ocul Pharmacol, 1994;10:439-51.

15. Goldblum D, Bachmann C, Tappeiner C, et al., Comparison of oral antiviral therapy with valacyclovir or acyclovir after penetrating keratoplasty for herpetic keratitis, Br J Ophthalmol, 2008:92:1201-5.

16. Tabbara KF, Al Balushi $\mathrm{N}$, Topical ganciclovir in the treatment of acute herpetic keratitis, Clin Ophthalmol, 2010;19;4:905-12.

17. Hoh HB, Hurley C, Claoue C, et al., Randomised trial of ganciclovir and acyclovir in the treatment of herpes simplex dendritic keratitis: a multicentre study, Br J Ophthalmol, 1996;80:140-3.

18. Tabbara KF, Treatment of herpetic keratitis, Ophthalmology, 2005;112:1640.

19. Tabbara KF, Pharmacologic strategies in the prevention and treatment of corneal transplant rejection, Int Ophthalmol, 2008;28:223-32

20. Wang X, Xu JJ, Le QH, et al., [Clinical assessment of oral ganciclovir capsules on the treatment of herpes simplex keratitisl, Zhonghua Yan Ke Za Zhi, 2010;46:994-9 (Article in Chinese).

21. Yanachkova M, Xu WC, Dvoskin $S$, et al., Prodrugs of herpes simplex thymidine kinase inhibitors, Antivir Chem Chemother 2015:24:47-55. 